\title{
MATHEMATICAL MODELING \& DYNAMICS STUDY OF BATCH CRYSTALLIZER FOR PURIFIED TEREPHTHALIC ACID (PTA) CRYSTALLIZATION PROCESS USING gPROMS
}

\author{
Abhishek A. Puttewar ${ }^{1}$, Surgonda T. Patil ${ }^{2}$ \\ ${ }^{1} P G$ Student, Department of Chemical Engineering, Tatyasaheb Kore Institute of Engineering \& Technology (TKIET) \\ Warananagar 416113, Dist: Kolhapur, Maharashtra, India \\ ${ }^{2}$ Associate Professor, Department of Chemical Engineering, Tatyasaheb Kore Institute of Engineering \& Technology \\ (TKIET) Warananagar 416113, Dist: Kolhapur, Maharashtra, India (Affiliated to Shivaji University, Kolhapur)
}

\begin{abstract}
Crystallization is a complex and critical chemical engineering process which is carried out for high purity products in process industry. Purified Terephthalic Acid (PTA) is commodity chemical, it is principal raw material mainly used in Polyester industry. During production process of Purified Terephthalic Acid (PTA), crystallization process is used to separate crystals from water. Crystalline materials generally have a high degree of purity even when obtained from a relatively impure solution. Control of crystal size distribution (CSD) is an end objective in PTA crystallization process. To fulfill CSD, needs the mathematical model based optimization for PTA unit, developed mathematical model is solved in gPROMS (general PROcess Modeling System).In present work, research achievement are batch crystallization modeling, simulation, optimization \& parameter estimation. Within the proposed control strategy, a dynamic optimization is performed to obtain optimal cooling temperature profile of batch crystallizer, maximizing the total volume of seeded crystals. Simulation is implemented to track the resulting optimal temperature profile.
\end{abstract}

Keywords: Purified Terephthalic Acid process, Mathematical model, Batch Crystallizer, simulation, gPROMS

\section{INTRODUCTION}

Crystallization is an important process, which is carried out for production of high purity in Terephthalic Acid industry. In the production process, oxidation and purification stages are carried out at high temperature and pressure, creating severe operating conditions. The process for purification can accept crude containing higher levels of impurities and still be able to produce suitable PTA. Efforts to purify PTA through crystallization using organic solvents have failed due to the inability to discover a solvent to economically purify by crystallization and a lack of understanding of the crystal growth mechanism. The effects of the process conditions and new crystallization method must be demonstrated at the pilot scale to stimulate industry investment. The common crystallization objective is to produce large crystals with minimum fines production by minimizing nucleation and optimizing the temperature profile. In batch cooling crystallization, the solution is cooled to super saturation causing crystal formation and its growth.

\section{MATHEMATICAL MODEL DEVELOPMENT}

\subsection{Population Balance Equation}

Mathematical representation of the crystallization rate can be achieved through basic mass transfer considerations or by writing a population balance represented by its moment equations. The population balance equation (PBE) is developed based on the following assumption: volume change in the system is negligible, crystal agglomeration or breakage phenomena are neglected and the crystal density and the supersaturation are homogeneous in all part of the crystallizer.

$$
\frac{\partial f\left(L_{s} t\right)}{\partial t}+G(t) \frac{\partial f\left(L_{s}, t\right)}{\partial L}=0
$$

The nucleation rate, $\mathrm{B}(\mathrm{t})$, and the growth rate, $\mathrm{G}(\mathrm{t})$ are [2], [4], [5], [7], [13] given by:

$$
\begin{gathered}
B(t)=k_{b} e^{-E b / R T}\left(\frac{C-C s(t)}{C s(t)}\right)^{b} \mu_{3}(t) \\
G(t)=k_{g} e^{-E b / R T}\left(\frac{C-C s(t)}{C s(t)}\right)^{g}
\end{gathered}
$$

\subsection{Model Implementation}

In the seeded batch crystallizer of PTA, the saturation concentration of the solute $\left(\mathrm{C}_{\mathrm{s}}\right)$ is given by

$$
C s=4.29 \times 10^{-6} e^{0.0392 T}
$$

By applying this moment operator to the population balance equation, a set of moment equation can be developed with the general form. 
The values of the model parameters of seeded batch crystallizer are shown in Table 1

\begin{tabular}{|l|l|l|}
\hline Symbol & Unit & Value \\
\hline $\mathrm{b}$ & - & 0.4719 \\
\hline $\mathrm{k}_{\mathrm{b}}$ & $\left(\mathrm{s} \mathrm{\mu m}^{3}\right)^{-1}$ & $6.5179 \times 10^{-12}$ \\
\hline $\mathrm{E}_{\mathrm{b}} / \mathrm{R}$ & $\mathrm{K}$ & $3.0068 \times 10^{3}$ \\
\hline $\mathrm{U}$ & $\mathrm{kJ}\left(\mathrm{m}^{2} \mathrm{~h} \mathrm{~K}\right)^{-1}$ & 1800 \\
\hline$\Delta \mathrm{H}_{\mathrm{c}}$ & $\mathrm{kJ} / \mathrm{Kg}$ & 122.7587 \\
\hline $\mathrm{M}$ & $\mathrm{kg}$ & 27.00 \\
\hline $\mathrm{K}_{\mathrm{v}}$ & - & 1.5 \\
\hline $\mathrm{Vj}$ & $\mathrm{m}^{3}$ & 0.015 \\
\hline$\rho \mathrm{j}$ & $\mathrm{Kg} / \mathrm{m}^{3}$ & 1000 \\
\hline $\mathrm{g}$ & - & 0.984 \\
\hline $\mathrm{kg}$ & $\mu \mathrm{m} / \mathrm{s}$ & 1.7251 \\
\hline $\mathrm{Eg} / \mathrm{R}$ & $\mathrm{K}$ & $1.7637 \times 10^{3}$ \\
\hline $\mathrm{A}$ & $\mathrm{m}^{2}$ & 0.25 \\
\hline $\mathrm{Cp}$ & $\mathrm{kJ}(\mathrm{kg} \mathrm{K})^{-1}$ & 3.86 \\
\hline$\rho_{\mathrm{c}}$ & $\mathrm{Kg} / \mathrm{m}^{3}$ & $1.58 \times 10^{-12}$ \\
\hline $\mathrm{T}_{\mathrm{f}}$ & $\mathrm{min}$ & 30 \\
\hline $\mathrm{Fj}$ & $\mathrm{m}^{3} / \mathrm{s}$ & 0.001 \\
\hline $\mathrm{Cp} p_{\mathrm{j}}$ & $\mathrm{kJ}(\mathrm{kg} \mathrm{K})^{-1}$ & 4.184 \\
\hline
\end{tabular}

Notations:

A is the total heat transfer surface area

$\mathrm{b}$ is an exponent relating nucleation rate to supersaturation

$\mathrm{C}$ is the solute concentration

$\mathrm{C}$ is the heat capacity of the solution

$\mathrm{C}^{\mathrm{p}}$ is the saturation concentration of the solute

$\mathrm{E}$ is the nucleation activation energy

$\mathrm{E}^{\mathrm{b}}$ is the growth activation energy

$f \stackrel{\mathrm{g}}{(L, t)}$ is the population density of crystals size $L$ at time $t$

$G(t)$ is the growth rate of crystals.

$\mathrm{F}$ is cooling water flow rate

$\mathrm{g}$ is an exponent relating growth rate to the supersaturation

$\mathrm{G}$ is crystallization growth rate

$\mathrm{k}$ is the volumetric shape factor

$\mathrm{M}$ is the mass of solvent is the crystallizer

$\mathrm{T}$ is the reactor temperature

$\mathrm{T}$ is the jacket temperature

$\mathrm{T}_{\text {jsp }}^{\mathrm{j}}$ is set point of the jacket temperature

$\mathrm{U}$ is the overall heat-transfer coefficient

$\mathrm{V}$ is jacket volume

$\rho$ is the density of the crystal

$\Delta \mathrm{H}$ is the heat of crystallization.

$\mu 3 \stackrel{c}{\text { is }}$ the third moment of the CSD

\subsection{Dynamic Optimization}

The aim of dynamic optimization is to maximize the average crystal size $(\mathrm{Lw})$ where to keep the coefficient of variations $\left(\mathrm{CV}_{\mathrm{w}}\right)$ small. The optimal cooling temperature profiles were obtained by solving the optimal control problem with different optimization problems.

\subsection{Simulation of model}

gPROMS is strongly typed modeling language for simulation and optimization of physical systems. Simulation models in gPROMS are defined using four different entities; DECLARE MODEL, TASK and PROCESS. The DECLARE entity is used to declare variables types and stream types, which will be used as templates for variables and streams. The model entity is use to describe the physical behavior of primitive elements of the system to be modeled.

Simulation result from gPROMS generated for Average Crystal Size (Lw) with respect to time (s)

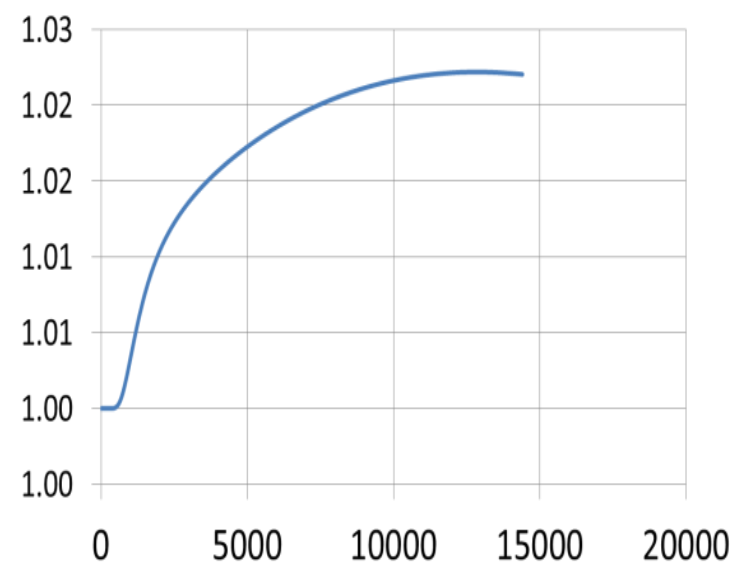

Fig 1: Lw Vs. T

Optimal profile for saturation concentration of solute $(\mathrm{Cs})$ is maintained with time

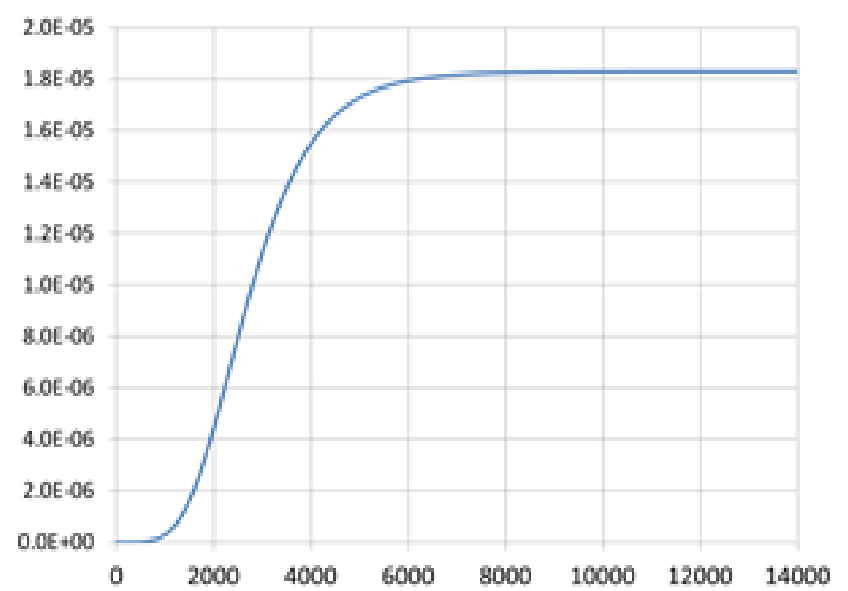

Fig 2 Cs Vs. T 
It is noted here that only the optimal cooling temperature profile obtained from selected optimized function in which the $\mu 3$ (tf) is minimized, is considered since such a temperature policy provides less fine crystals leading to efficient operation in downstream processes whereas the volume of seeded crystal $(\mu 3)$ is still satisfy the product quality requirement.

Output of Simulation result shows with different Liquid Volume in the same vessel does not matter to crystal size

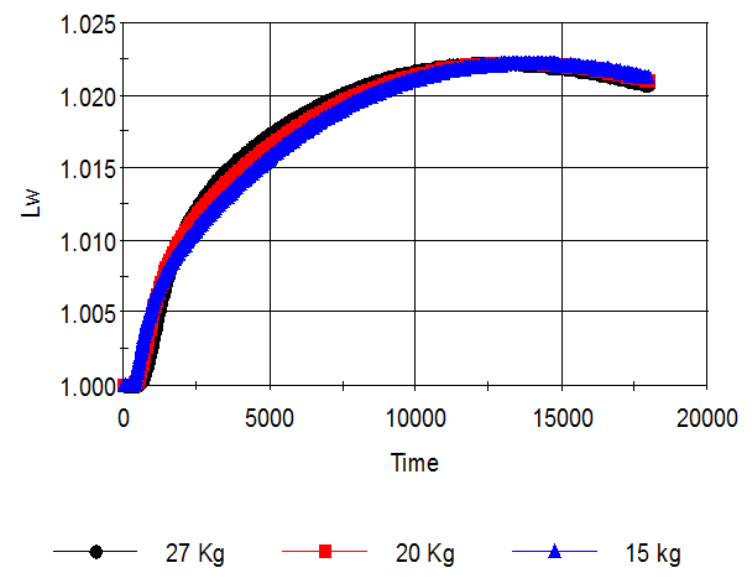

Fig 3: Effect of liquid volume on Lw Vs. T

Output of simulation shows different concentration of solids are maintained with respect to time However smaller liquid volumes help faster growth of crystals.

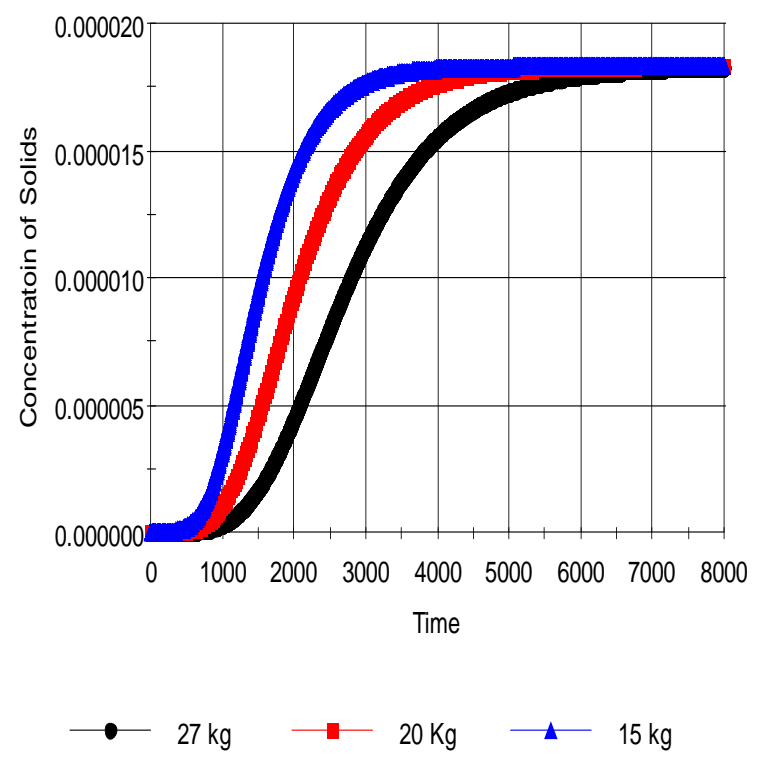

Fig 4: Effect of liquid volume on Cs Vs Time

Output shows Increase in overall heat transfer coefficient (U) causes faster heat transfer leading to faster crystallization as observed.

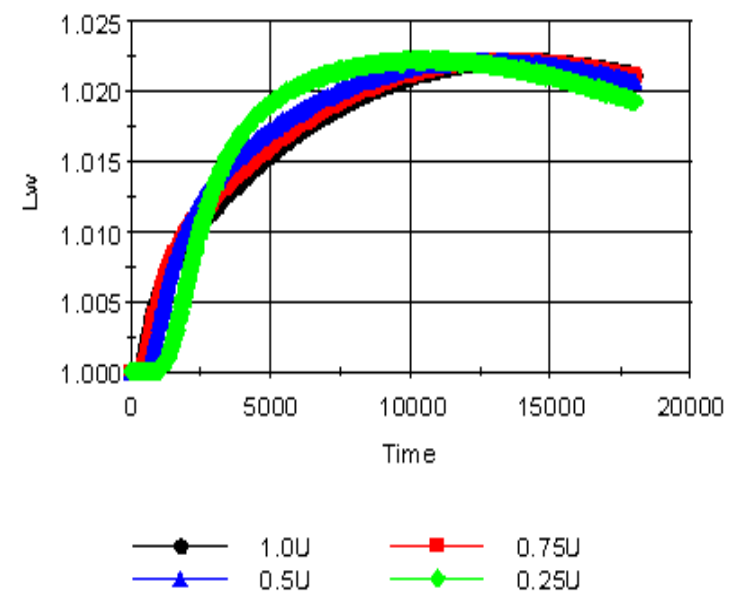

Fig 5: Effect of U on Lw Vs. T

Output of Simulation result shows, Average crystal size decreases with lesser $\mathrm{U}$ owing to longer heater transfer time.

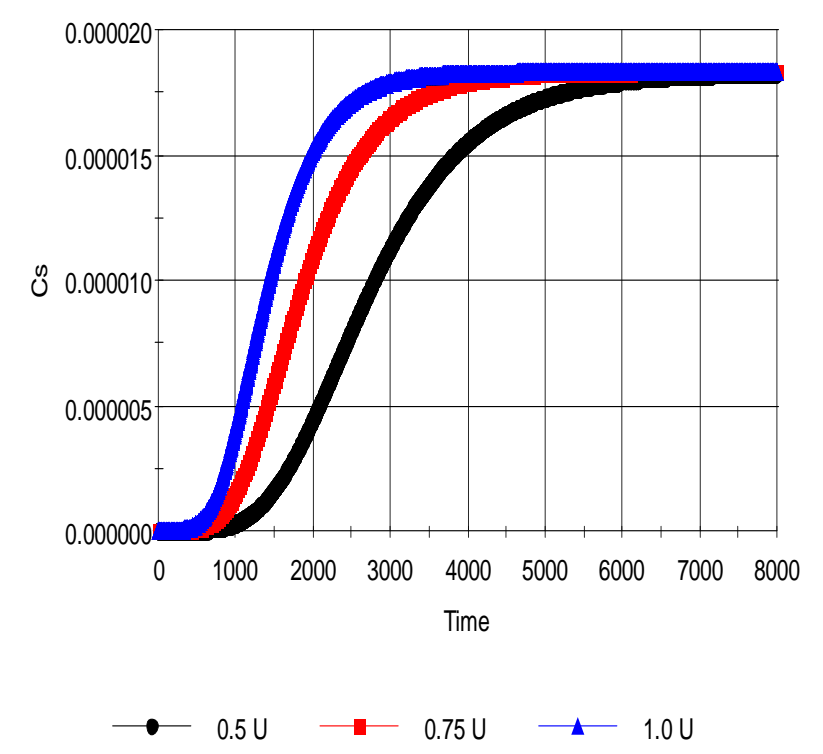

Fig 6: Effect of change of U on Cs Vs. T Output of simulation for Moment of CSD against time, with change in heat transfer co-efficient 


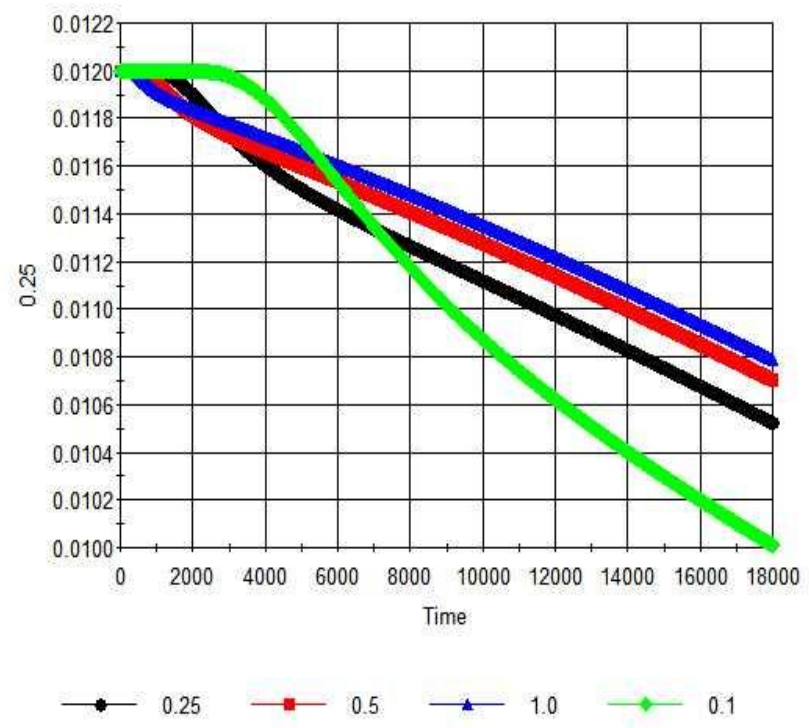

Fig 7: Effect of change of $U$ on $\mu$ and Time

Output of simulation for Moment of CSD against time, with change in heat transfer co-efficient
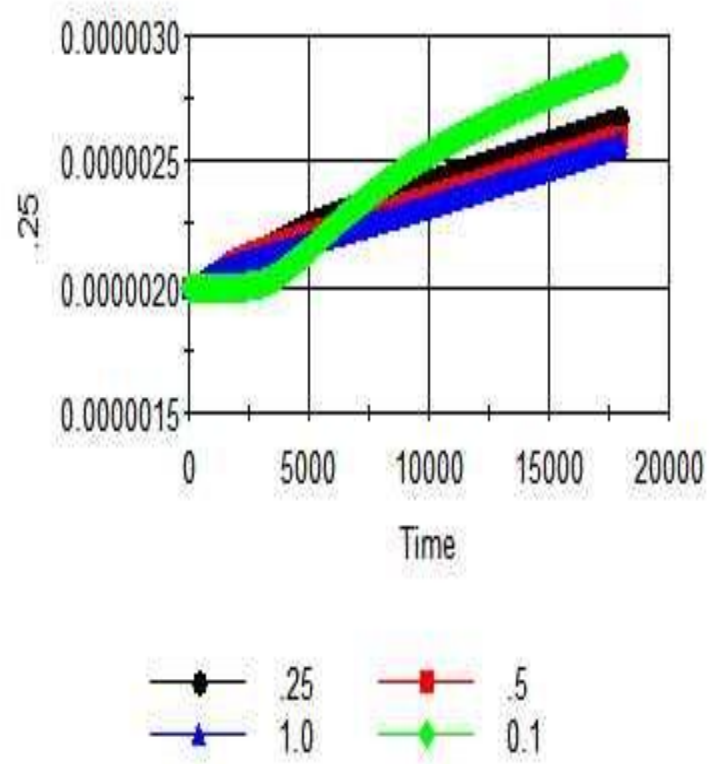

Fig 8: Effect of change of $U$ on Temperature \&Time

Output of simulation for Moment of CSD against time, with change in heat transfer co-efficient

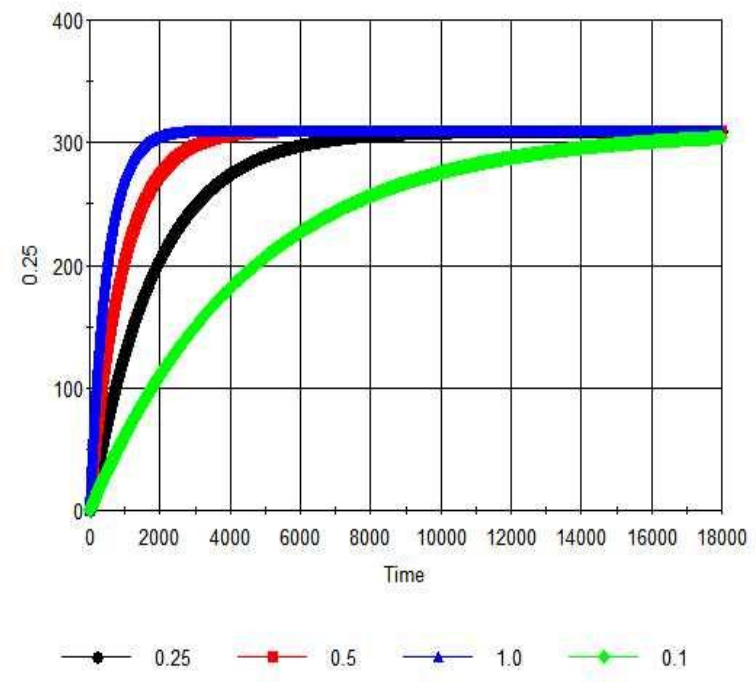

Fig 9: Effect of change of U on Lw and Time

\section{PTA BATCH CRYSTALLIZER GPROMS PROGRAM CODE:}

\section{Model Crystallizer}

PARAMETER

\#exponent relating nucleation rate to the supersaturation b AS REAL

kb AS REAL

\#the nucleation activation energy

EbbyR AS REAL

\#the overall heat-transfer coefficient

U AS REAL

\#the heat of crystallization.

dHc AS REAL

\#the mass of solvent is the crystallizer

M AS REAL

\#the volumetric shape factor

Kv AS REAL

\#jacket volume

Vj AS REAL

\# the density of jacket fluid rhoj AS REAL

\# an exponent relating growth rate to the supersaturation g AS REAL

kg AS REAL

\# the growth activation energy

EgbyR AS REAL 
\# total heat transfer surface area

A AS REAL

\# the heat capacity of the solution

Cp AS REAL

\# the density of the crystal

rhoc AS REAL

\# Tf in min

Tf AS REAL

\# cooling water flow rate

Fj AS REAL

\#the heat capacity of jacket fluid

Cpj AS REAL

mu0s AS REAL

\section{VARIABLE}

\# the saturation concentration of the solute (Cs)

Cs AS Concentration

\# the solute concentration

C AS Concentration

\# Crystalliser Temperature

T AS Temperature

\# Jacket Temperature

Tj AS Temperature

\# set point of the jacket temperature

Tjsp AS Temperature

\# the growth rate of crystals

Gt AS NoType

\#the ith moment of the CSD that explains the total volume

of crystals

mu0n, mu1n, mu2n, mu3n, mu4n,mu5n AS NoType

mu1s, mu2s, mu3s, mu4s, mu5s AS NoType

mu0, mu1, mu2, mu3,mu4,mu5 AS NoType

\# The nucleation rate

Bt AS NoType

\# Average crystal size

Lw AS NoType

\#CVw coefficient of variations

CVw AS NoType

DISTRIBUTION_DOMAIN

SET

BOUNDARY

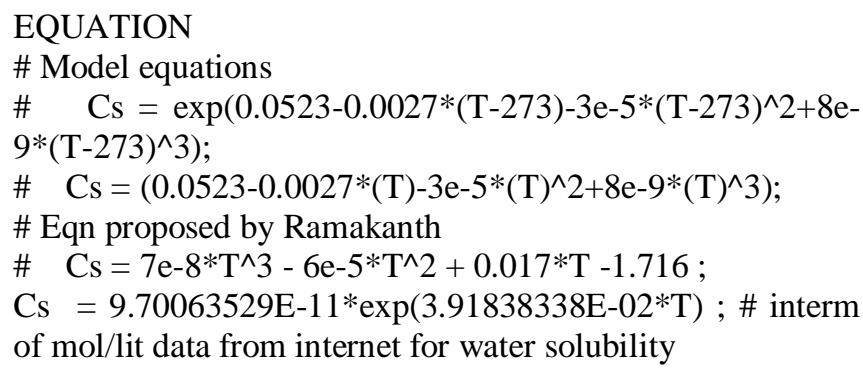

UNIT \# Equipment items

\# AP101 AS Crystalliser

AP101 AS Crystallisation

SET

\# Parameter values

\# exponent relating nucleation rate to the supersaturation AP101.b:=0.4719;

\#coeff relating nucleation rate to the supersaturation um/s AP101.kb:= 6.5179e-12; 
\#the nucleation activation energy

AP101.EbbyR:= 3.0068e+3;

\#the overall heat-transfer coefficient $\mathrm{kJ} / \mathrm{m} 2 \mathrm{sK}$

AP101.U:=0.5; \# =1800 kJ/(m2 h K $)$

\#the heat of crystallization.kj/kg

AP101.dHc:= 122.7587;

\#the mass of solvent is the crystallizer in $\mathrm{kg}$

AP101.M:= 27.0;

\#the volumetric shape factor constant

AP101.Kv:=1.5;

\#jacket volume in $\mathrm{m} 3$

AP101.Vj:=0.015;

\# the density of jacket fluid $\mathrm{kg} / \mathrm{m} 3$

AP101.rhoj:= 1000;

\# an exponent relating growth rate to the supersaturation AP101.g:=0.984;

\# ancoeff relating growth rate to the supersaturation $\mathrm{m} / \mathrm{s}$ AP101.kg:= 1.7251e-6;

\# the growth activation energy $\mathrm{K}$

AP101.EgbyR:=1.7637e3;

\# total heat transfer surface area $\mathrm{m} 2$

AP101.A: $=0.25$;

\# the heat capacity of the solution $\mathrm{kJ} /(\mathrm{kg} \mathrm{K})$

AP101.Cp:=3.86;

\# the density of the crystal $\mathrm{kg} / \mathrm{m} 3$ Orig $=1.58 \mathrm{e} 12$

AP101.rhoc:= 1.58E3;

\# Tf in sec

AP101.Tf: $=30 * 60$;

\# cooling water flow rate $\mathrm{m} 3 / \mathrm{s}$

AP101.Fj:=0.001;

\#the heat capacity of jacket fluid $\mathrm{kJ} /(\mathrm{kg} \mathrm{K})$

AP101.Cpj:=4.184;

AP101.mu0s: $=0.0$;

\# condition

ASSIGN

WITHIN AP101 DO

Tjsp: $=310$;

END

INITIAL

WITHIN AP101 DO

$\mathrm{C}=0.012$;

$\mathrm{Tj}=303.15$;
\# $\mathrm{T}=500$;

mu0n =1e-6;

muln $=1.0 \mathrm{e}-6$;

$\mathrm{mu} 2 \mathrm{n}=1.0 \mathrm{e}-6$

mu3n $=1.0 \mathrm{e}-6$

$\mathrm{mu} 4 \mathrm{n}=1.0 \mathrm{e}-6$

mu5n $=1.0 \mathrm{e}-4$

$\mathrm{mu} 2 \mathrm{~s}=1.0 \mathrm{e}-6$;

$\mathrm{mu} 3 \mathrm{~s}=1.0 \mathrm{e}-6$;

$\mathrm{mu} 4 \mathrm{~s}=1.0 \mathrm{e}-6$;

$\mathrm{mu} 5 \mathrm{~s}=1.0 \mathrm{e}-6$

$\$ \mathrm{mu} 0 \mathrm{n}=10 \mathrm{e}-6$

$\$ \mathrm{mu} 2 \mathrm{~s}=10 \mathrm{e}-6$;

\# $\quad \mathrm{CvW}=0$;

END \# Within AP101

SOLUTIONPARAMETERS

Reporting Interval $:=1$;

SCHEDULE

CONTINUE FOR $5 * 3600$

\section{CONCLUSIONS}

From the above studies the following conclusions are made

1. Mathematical Modelling is developed for batch crystallizer

2. Developed mathematical model is solved in gPROMS

3. Model is used to predict the concentration and crystallizer temperature profile in this process

4. Optimal temperature profile for best Crystal size distribution (CSD)

5. In seeded batch crystallization process, large average crystal size leading to product quality is desired.

\section{ACKNOWLEDGEMENTS}

Author would like to acknowledge the support from my family and the department of Chemical Engineering, Faculty of Chemical Engineering, TKIET Warananagar and Shivaji University, Kolhapur.

\section{REFERENCES}

[1] David Widenski, Ali Abbas, Jose Ramagnoli, A theoretical nucleation study of the combined effect of seeding and temperature profile in cooling crystallization, $10^{\text {th }}$ International symposium on Process Systems Engineering, PSE2009, Elsevier Publication, pages 423-430.

Ian T. Cameron, Rafiqul Gani, Product and Process Modelling: A case study Approach, Elsevier Publication, Page 305-336.

[3] Lin Niu, Dongyue Yang, Dynamic Simulation and optimization for Batch Reactor Control Profiles, $4^{\text {th }}$ International Conference on Computer Modelling and Simulation (ICCMS 2012).IPCSIT vol.22 (2012), Page 58-61.

[4] Petia Georgieva, and Sebastião Feyo de Azevedo, Application of Feed Forward Neural Network in Modelling and Control of a Fed -Batch 
Crystallization Process, Proceedings of world academy of science, Engineering and Technology, Vol.12, March 2006, Pages 65-70.

[5] Q.Hu, S.Rohani, D.X.Wang and A.Jutan, Optimal control of batch cooling seeded crystallizer, Powder Technology, Vol 156,2005, Page 170-176

[6] W.Paegjuntuek, A.Arpornwichanop, and P. Kittisupakorn, Product quality improvement of batch crystallisers by a batch-to-batch optimization and nonlinear control approach, Chemical Engineering Journal, Vol 139,2008, Page 344-350

[7] Preeya Somsong, Paisan Kittisupakorn, Kasidit Nootong, Wachira Daosud, Neural Network Modeling and Optimization for a Batch Crystallizer to Produce Purified Terephthalic Acid, The First International Conference on Interdisciplinary Research and Development, 31 May - 1 June 2011, Thailand, Pages8.1-8.5.

[8] K.L.Choong, R.Smith, Optimization of batch cooling crystallization, Elsevier Publication, Chemical Engineering Science 59 (2004), Pages 313-327

[9] Wenli Du, Feng Qian, Optimization of PTA crystallization process based on fuzzy GMDH networks and differential evolutionary Algorithm, ICNC 2005, LNCS 3611, 2005, Pages 631 -635

[10] Paul A.Larsen, Daniel B. Patience, James B. Rawlings, Industrial Crystallization Process control, IEEE Control system magazine, August 2006, Page 70-80

[11] W. Wohlk, G. Hofmann, Types of Crystallizers, International Chemical Engineering, Vol.27, No.2, April 1987, Pages 197-204

[12] Process Systems Enterprise Ltd, gPROMS Introductory User guide course, 2004

[13] Process Systems Enterprise Ltd, gPROMS Advanced Used guide course, 2004

[14] Mu Shengjing, Su Hongye, Gu Yong, Chu Jian, Multi-Objective Optimization of Industrial Purified Terephthalic Acid Oxidation process, Chinese Journal of Chemical Engineering, Vol 11 (5), 2003, Page 536-541

[15] S.H Chung, D.L.Ma and R.D.Braatz, Optimal Seeding in batch crystallization, Canadian Journal Chemical Engineering, Vol. 77 No.3,1999, Page $590-596$

[16] Gregg T.Beckham, Baron Peters, Cindy Starbuck, Narayan Variankaral and Bernhardt L. Tront,Surface-mediated nucleation in the solid-state polymorph transformation of Terephthalic acid, Journal of American Chemical Society, 2007, Page A1-J10

[17] Ketan Samant and Lionel O.Young, Understanding Crystallization and Crystallizers, AIChe, October 2006, Page 28-36

[18] Sang Jo Kim and Se Young koh, Samsung Petrochemical Company, Orbit, May 1992, Page 1520
[19] Sung Hwa Jhung and Youn-Seol Park, Hydropurification of Crude Terephthalic Acid over $\mathrm{PdRu} /$ Carbon composite catalyst, Journal of the Korean Chemical Society, Vol.46 No.1, 2002, Page 57-63

[20] Dong-Koo Lee, Tae-sun Chang and Chae-Ho Shin, Thermal Treatment of Crude Terephthalic Acid recovery from Alkali weight reduction wastewater, Journal Ind.Eng.Chem., Vol.8 No.5, 2002, Page 405-409

[21] Li Chengfei, Dynamic Simulation and Analysis of Industrial Purified Terephthalic Acid Solvent Dehydration Process, Chinese Journal of Chemical Engineering, vol.19, 2011, Page 86-96

[22] Naim Bajcinca, Steffan Hofman, Optimal Control for Batch Crystallization with size-dependent growth kinetics, American Control Conference, 2011, Page 2558-2565

[23] Sean K.Bermingham, Herman J.M.Kramer, Peter J.T.Verheijen, Experience with Dynamic Modelling of Crystallization systems, NPT Procestechnologie, 2004, Page 16-19

[24] Zoltan K.Nagy, A Population Balance model approach for Crystallization product engineering via distribution shaping control, $18^{\text {th }}$ European Symposium on Computer Aided Process Engineering, 2008, Page 1-6

[25] Yataro Ichikawa, Gentaro Yamashita, Michiyuki Tokashiki \& Teizo Yamaji, New Oxidation Process for Production of Terephthalic Acid from p-Xylene, European \& Japanese Chemical Industries Symposium, Vol. 62 No.4, April 1970, Page 38-42

\section{BIOGRAPHIES}

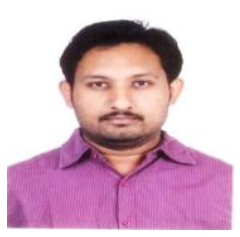

Abhishek A. Puttewar received B.E. (Chemical Engineering) degree from Shivaji University; Kolhapur (Padmabhushan Vasantraoadada Patil Institute of Technology, Budhagaon, Sangli) in 2008.He is currently M.E. (Chemical Engineering) student in Tatyasaheb Kore Institute of Engineering \& Technology, Warananagar from Shivaji University Kolhapur. His research interest includes modeling \& simulation

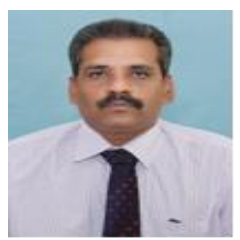

Surgonda T. Patil obtained his B.E. and M.E. in Chemical Engineering from Shivaji University, Kolhapur, India. He has 22 years of teaching experience. His interests include process calculations, modeling \& simulation in chemical engineering Presently he is Associate Professor in Chemical Engineering Department of TKIET, Warananagar. Kolhapur, India. 